\title{
Proposal of Classification of Aneurysms Coexisting with AVM and Possible Treatment Strategies
}

\author{
Xianli LV, Zhongxue WU, Hongwei HE, Huijian GE, Youxiang LI \\ Capital Medical University, Beijing Neurosurgical Institute and Beijing Tiantan Hospital, Interventional Neuroradiology Department, \\ Beijing, China
}

\section{ABSTRACT}

AIM: To describe the classification of aneurysms coexisting with arteriovenous malformations (AVMs) and their endovascular treatment.

MATERIAL and METHODS: Based on the basis of radiological findings and clinical features, 59 aneurysms coexisting with AVMs were classified into four different types: intranidal, flow-related (proximal and distal), and unrelated. These aneurysms were treated by endovascular techniques with or without AVM embolization. Clinical outcome was assessed using the modified Rankin Scale (mRS).

RESULTS: 59 aneurysms coexisting with AVMs were found in 39 patients. There were 11 unrelated aneurysms, 18 proximal flowrelated aneurysms, 11 distal flow-related aneurysms and 19 intranidal aneurysms. In the 11 unrelated aneurysms, 4 were caused subarachnoid hemorrhage. In 11 distal flow-related aneurysms near the AVM, 7 caused a major hemorrhage. Six of the 19 intranidal aneurysms presented with hemorrhage. In contrast, only 3 of the 14 proximal flow-related aneurysms on major feeding arteries caused hemorrhage.

CONCLUSION: Aneurysms coexisting with AVMs could be classified into four types and this classification may provide a rationale for endovascular treatment.

KEYWORDS: Aneurysm, Arteriovenous malformation, Endovascular treatment

\section{INTRODUCTION}

Internal elastic lamina (IEL) damage and low shear stress are often risk factors in the pathogenesis of aneurysm formation $(6,22)$. The incidence of aneurysms coexisting with AVMs is well documented to be between $10 \%$ and $58 \%(7,25,29)$. The aneurysms coexisting with AVMs can be divided into four groups: unrelated dysplastic or incidental (Type 1); flowrelated on proximal feeding vessels (Type 2); flow-related on distal small feeding vessels (Type 3); and intranidal (Type 4) $(5,22)$. There is still confusion as to the etiology and clinical and radiological features of aneurysms coexisting with AVMs. Some authors have traditionally regarded associated aneurysms as independent risk factors and treated them aggressively $(10,17)$. In the present study, we propose a classification system that unifies aneurysms coexisting with AVMs and their endovascular treatments (Table I).

\section{MATERIAL and METHODS}

A series of 111 consecutive patients with cerebral AVMs between February 2009 and February 2011 were prospectively reviewed. Among these patients, 39 (35.1\%) patients were diagnosed with AVMs and accompanying intracranial arterial aneurysms. Diagnosis was based on clinical presentations and radiologic findings, including computed tomography (CT) scanning, magnetic resonance imaging (MRI), and cerebral angiography. We attempted to classify the aneurysms on 
Lv X. et al: Classification of Aneurysms Coexisting with AVM

Table I: Summary of Types of Aneurysms Coexisting with AVM

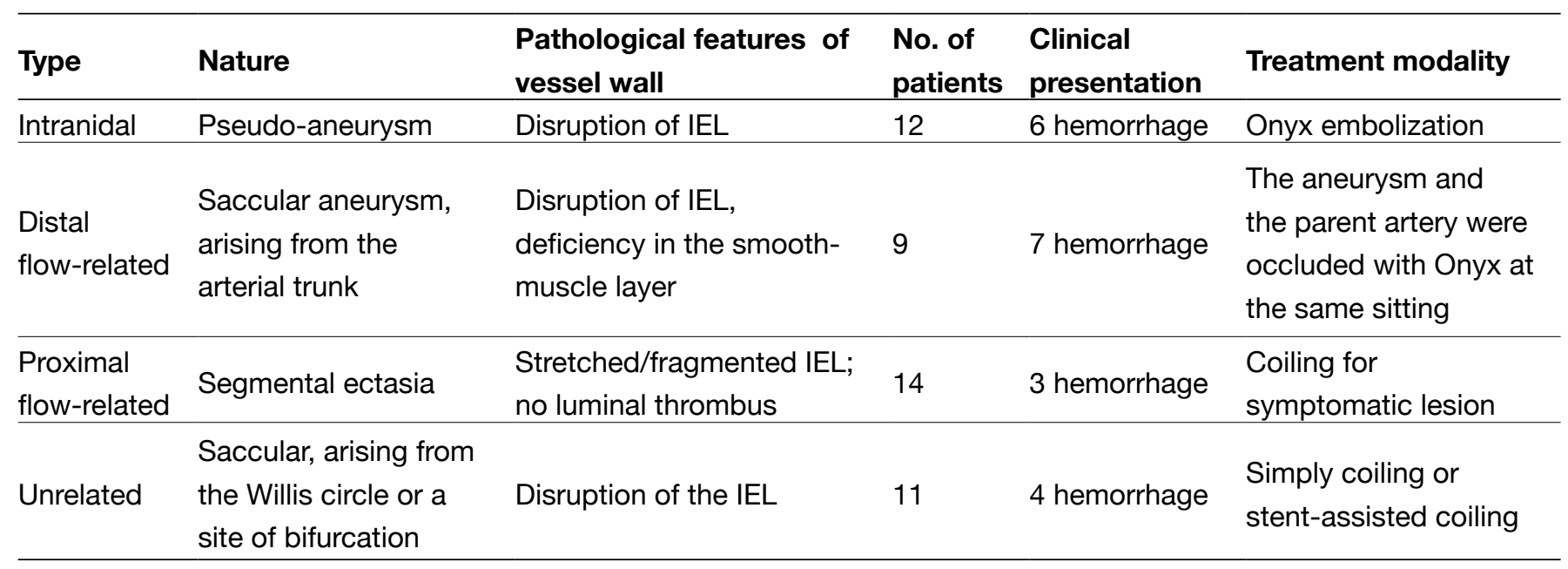

IEL: Internal elastic lamina.

the basis of radiological findings and clinical features. The outcome was assessed using the modified Rankin Scale $(\mathrm{mRS})$.

\section{Classification of Aneurysms}

Aneurysms arising from the proximal arterial trunk supplying the AVM were classified as proximal flow-related aneurysms and unrelated aneurysms were located on the Willis circle or a site of bifurcation. Aneurysms arising from the distal small feeding vessels were classified as distal flow-related aneurysms and those in the immediate vicinity of the AVM nidus or within the AVM nidus were classified as intranidal aneurysms.

\section{Treatment Strategies}

1. Distal flow-related and intranidal aneurysms were treated in conjunction with AVM Onyx embolization.

2. Unruptured proximal flow-related aneurysms were followed and ruptured ones were treated with coiling.

3. Unrelated aneurysms (arising from Willis circle or a site of bifurcation) were treated with coiling.

\section{RESULTS}

\section{Presentations}

Of the 39 patients, there were 12 men and 27 women and the age range was from 5 to 72 years (mean, 34.4 years). Twentyfour $(61.5 \%)$ patients initially presented with hemorrhage. The remaining 15 patients (38.5\%) initially presented with symptoms or signs unrelated to hemorrhage. Eight $(20.5 \%)$ AVMs were infratentorial and 31 (79.5\%) were supratentorial.

\section{Aneurysm Characteristics}

These patients harbored 59 coexisting aneurysms. Fifteen patients had AVMs with multiple arterial aneurysms. There were 11 unrelated aneurysms, 18 proximal flow-related aneurysms, 11 distal flow-related aneurysms and 19 intranidal aneurysms.
Four of the 11 unrelated aneurysms caused subarachnoid hemorrhage. Seven of the 9 distal flow-related aneurysms near the AVM caused a major hemorrhage, which was evident on angiogram. Six of 19 intranidal aneurysms presented with hemorrhage. In contrast, only 3 of 14 proximal flow-related aneurysms on major feeding arteries caused hemorrhage, which was identifiable as a source of bleeding on CT scanning.

\section{Unrelated Aneurysm}

Among the 11 unrelated aneurysms, 3 were in the anterior communicating artery (Figure 1A-C), 4 in the internal carotid artery-posterior communicating artery, 2 at the basilar tip, 1 in the vertebral artery-anterior inferior cerebellar artery, and 1 in the middle cerebral artery bifurcation. All these aneurysms were small saccular aneurysms ( 3 to $7 \mathrm{~mm}$ ) with a small neck.

\section{Proximal Flow-Related Aneurysms}

Among the 18 proximal flow-related aneurysms, 8 on the internal carotid artery were very small saccular aneurysms ( $\leq 3 \mathrm{~mm}$ ), 3 on the middle cerebral artery were small fusiform aneurysms ( $\leq 4 \mathrm{~mm}), 2$ on the anterior cerebral artery were small fusiform aneurysm ( $\leq 4 \mathrm{~mm}), 1$ on the superior cerebellar artery was a small fusiform aneurysm $(5 \mathrm{~mm}), 2$ on the thalamus perforating artery pedicle were small fusiform aneurysm ( 5 and $6 \mathrm{~mm}), 1$ on the anterior cerebellar artery was a small fusiform aneurysm $(5 \mathrm{~mm})$ and 1 on the posterior cerebral artery was a small fusiform aneurysm $(8 \mathrm{~mm})$.

\section{Distal Flow-Related Aneurysms}

The 11 distal flow-related aneurysms, and 2 middle cerebral artery, 3 anterior choroidal artery, 3 posterior inferior cerebellar artery, 1 middle cerebral artery, 1 anterior cerebral artery and 1 superior cerebellar artery aneurysms, were small saccular aneurysms.

\section{Intranidal Aneurysms}

All 19 intranidal aneurysms were of the saccular type (5 to 12 $\mathrm{mm})$. 


\section{Aneurysm Treatment}

All unrelated aneurysms were treated with coiling, and distal flow-related and intranidal aneurysms were treated in conjunction with AVM Onyx embolization (Figure 1A-C). For proximal flow-related aneurysms, only 3 ruptured cases were coiled and the other 15 were left untreated. In 1 patient, a proximal flow-related aneurysm on the feeding vessel of the anterior cerebellar artery, which was left untreated, led to hemorrhage 2 months later and it was coiled.

\section{AVM Treatment}

Forty-one Onyx embolization procedures were performed for AVM nidus creating a new or worsening a preexisting neurological deficit in 6 patients and slowly resolving paralysis and dysarthria in 2 patients. Additional gamma knife radiosurgery was performed in 6 cases. Complete obliteration of AVMs was achieved in 6 patients (15.4\%). During a 17-month (9-23 months) follow-up period, 30 (76.9\%) made a good recovery ( $\mathrm{mRS} 0)$ and $9(23.1 \%)$ had a moderate disability (mRS $1-3)$ related to hemorrhage.

\section{DISCUSSION}

\section{Classification}

Meisel et al. (21) reported on 305 AVMs associated with aneurysms and suggested that intranidal aneurysms should be primary treatment targets whereas proximal aneurysms should not. Their rationale was based on evidence that the latter often regress after definitive AVM treatment and on their finding that proximal aneurysms were not associated with the same increase in hemorrhage rate as intranidal aneurysms. Redekop et al. (26) and Meisel et al. (21) presented good evidence for the treatment of intranidal aneurysms and observation of proximal aneurysms. Since flow-related aneurysms may well be located on the circle of Willis, it should be defined that they refer to unrelated aneurysms when talking about circle of Willis aneurysms. Others have summarized distal and intranidal aneurysms together, mixing two very different entities. Although some authors recently started to use this terminology, it is still not a good definition. Classification to intranidal, flow-related (proximal and distal), and unrelated is better terminology. This is important since unrelated aneurysms constitute a different type of pathology as compared to flow-related or especially intranidal ones, with different rupture risk and thus requiring a different therapeutic approach.

\section{Pathogenesis of These Aneurysms}

Aneurysm formation may be the result of a break in the equilibrium between hemodynamic stress and the condition of the IEL and the intima (22). Hemodynamic stress is presumed to be the primary factor causing remodeling, degeneration, and loss of the IEL. Intranidal aneurysms should be considered "false aneurysms" caused by nidus rupture rather than aneurysm rupture, with acute disruption of the IEL, a false lumen, and a high rate of recurrent hemorrhage. Proximal flow-related aneurysms are simple ectatic arteries with benign clinical behavior; the IEL is stretched or fragmented, and the luminal surface of the intima is smooth. Therefore, proximal flow-related aneurysm is a dilated segment of artery without IEL disruption and has a benign course. Because small arteries involved in arteriovenous malformation are deficient in the smooth-muscle layer (18), the distal flow-related aneurysm lacks IEL and smooth-muscle layer in its dome and may rebleed easily owing to the fragile aneurysmal wall. Unrelated aneurysms (saccular aneurysms arising at arterial bifurcations) are assumed to be acquired lesions caused by a combination of hemodynamic stresses (luminal factors) and defective vessel wall responses (abluminal factors) (11). AVMs coexisting with aneurysms are significantly associated with hemorrhage, older age, and infratentorial AVM location $(3,14,30)$. Exogenous factors, such as cigarette smoking
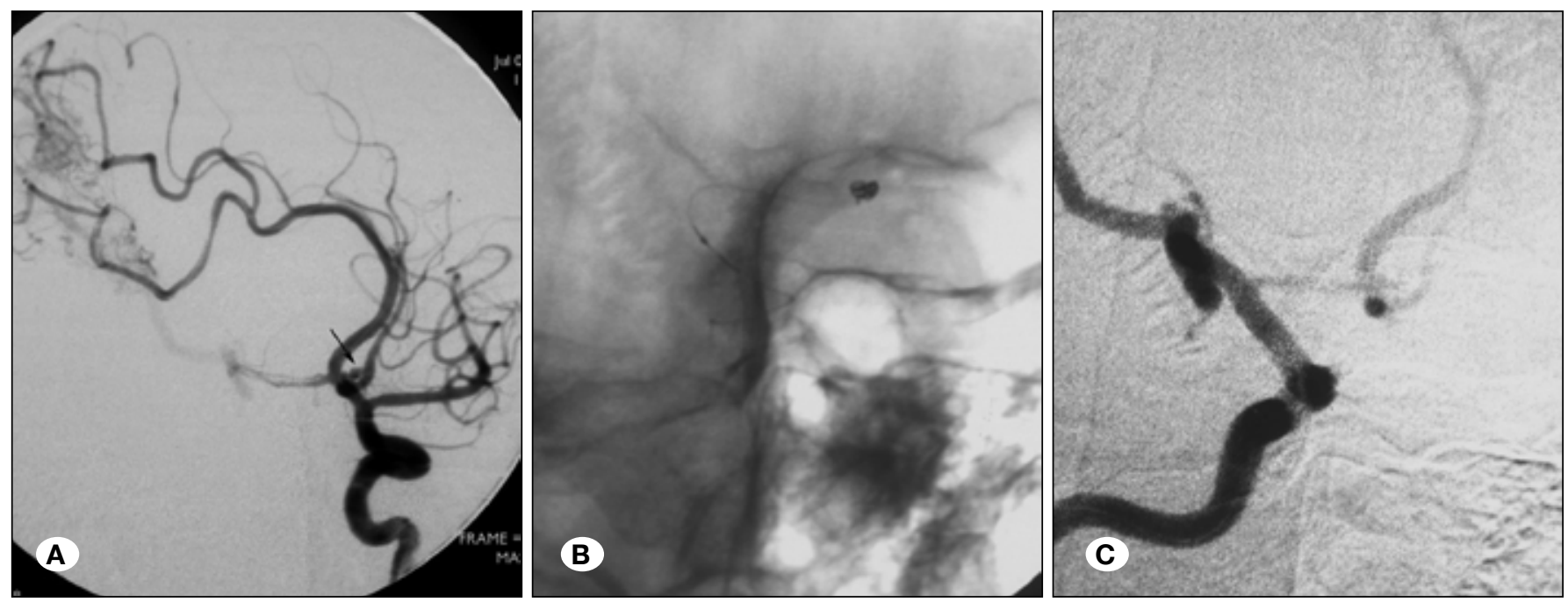

Figure 1: A) Cerebral arteriogram after injection of the left internal carotid artery with contrast medium shows an unrelated saccular aneurysm on the anterior communicating artery. B) The aneurysm was treated with coil embolization. C) Angiogram of the left internal carotid artery shows that th e aneurysm was completely obliterated. 
and alcohol consumption, are similarly associated with the formation and rupture of classic saccular aneurysms. In contrast to recent experimental reports of the association between statins and a reduction of intracranial aneurysm formation, the findings of Marbacher et al. (19) suggest that statins may have no significant beneficial effect on intracranial aneurysm suppression in humans.

\section{Risk Attributable to Coexisting Aneurysms}

The annual bleeding risk of an untreated AVM is $2 \%-3 \%$ per year and the risk of recurrent hemorrhage is even greater. The risk of death with an AVM hemorrhage is approximately $10 \%$, and the risk of a neurologic deficit is approximately $50 \%$ (27). After an initial hemorrhage, the annual risk of a subsequent hemorrhage has been reported to range from 4.5 to $34.4 \%$ $(4,9,20,24,28)$; the best estimate is approximately $6 \%$ during the first year, with a return to the baseline risk afterward $(8,31)$. It is also well demonstrated that an additional bleeding risk is present in AVMs coexisting with aneurysm (15). Redekop et al. (26) reported a $10 \%$ risk of hemorrhage per year in patients with intranidal aneurysms, which is considerably greater than the $2 \%-4 \%$ annual hemorrhage rate usually attributed to AVMs.

\section{Subtypes of These Aneurysms Associated with the Greatest Risk of Hemorrhage}

Arterial aneurysms may occur in any segment of the feeding arteries and the larger the aneurysm, the greater the risk of rupture $(2,3,12)$. We $(16)$ have retrospectively reviewed 302 patients treated with AVMs at our institution. In that series, intranidal aneurysms were present in $13.6 \%$ of cases, and extranidal aneurysms were present in $10.9 \%$. Willis Circle aneurysms were managed similar to the general aneurysms. We found that coexisting aneurysms more often became symptomatic with hemorrhage, in older patients, and in the case of infratentorial AVMs. In our previous series, Willis circle aneurysms were associated with significantly more hemorrhage than that of flow-related aneurysms. Willis circle aneurysms also had a greater incidence of subarachnoid hemorrhage and should be treated primarily. Furthermore in this series, 4/11 arterial bifurcation unrelated aneurysms, $7 / 9$ distal flow-related aneurysms near the AVM and 6/12 intranidal aneurysms caused hemorrhage. However, only $3 / 14$ proximal flow-related aneurysms on major feeding arteries caused hemorrhage. The risk of hemorrhage from a proximal flow-related aneurysm that has not bled seems to be lower than that of other aneurysms and it should not be assumed that all cases need treatment.

\section{Treatment Modality}

Although feeding-artery aneurysms less than $5 \mathrm{~mm}$ in diameter have been reported to regress after treatment of AVM in some cases (12), they have ruptured during/after AVM treatment like in one of our patients, probably owing to the arterial bifurcation type, in other cases. Given the concern about possible rupture, endovascular coiling of the arterial bifurcation aneurysm before AVM embolization should be performed $(23,26)$. The distal flow-related aneurysm is often wide-necked, and may be difficult to clip and one can be directed to the lesion using Onyx embolization (13). Spetzler et al. have advocated that Grade IV and V AVMs be managed conservatively (1). However, in the presence of associated aneurysms, we believe that the aneurysm should be treated with or without intervention to the AVM.

\section{CONCLUSION}

Aneurysms coexisting with AVMs could be classified into four types and this classification may provide a rationale for endovascular treatment. Unrelated, distal flow-related and intranidal aneurysms have a high rate of hemorrhage and should be treated. The asymptomatic proximal flow-related aneurysm may not require treatment.

\section{REFERENCES}

1. Almefty K, Spetzler RF: Arteriovenous malformations and associated aneurysms. World Neurosurg 76:396-397, 2011

2. Batjer H, Suss RA, Samson D: Intracranial arteriovenous malformations associated with aneurysms. Neurosurgery 18:2935,1986

3. Brown RD Jr, Wiebers DO, Forbes GS: Unruptured intracranial aneurysms and arteriovenous malformations: Frequency of intracranial hemorrhage and relationship of lesions. J Neurosurg 73:859-863, 1990

4. Crawford PM, West CR, Chadwick DW, Shaw MD: Arteriovenous malformations of the brain: Natural history in unoperated patients. J Neurol Neurosurg Psychiatry 49:1-10, 1986

5. de los Reyes RA, Fink ME: Special problem: AVM associated with aneurysm. In: Batjer $\mathrm{HH}$ (ed). Cerebrovascular Disease. Philadelphia: Lippincott-Raven, 1997:743-747

6. Doenitz C, Schebesch KM, Zoephel R, Brawanski A: A mechanism for the rapid development of intracranial aneurysms: A case study. Neurosurgery 67:1213-1221, 2010

7. Friedlander RM: Arteriovenous malformations of the brain. N Engl J Med 356:2704-2712, 2007

8. Graf CJ, Perret GE, Torner JC: Bleeding from cerebral arteriovenous malformations as part of their natural history. J Neurosurg 58:331-337, 1983

9. Hartmann A, Mast H, Mohr JP, Koennecke HC, Osipov A, PileSpellman J, Duong DH, Young WL: Morbidity of intracranial hemorrhage in patients with cerebral arteriovenous malformation. Stroke 29:931-934, 1998

10. Krings T, Hans FJ, Geibprasert S, Terbrugge K: Partial "targeted" embolisation of brain arteriovenous malformations. Eur Radiol 20:2723-2731, 2010

11. Krings T, Mandell DM, Kiehl TR, Geibprasert S, Tymianski M, Alvarez $\mathrm{H}$, terBrugge KG, Hans FJ: Intracranial aneurysms: From vessel wall pathology to therapeutic approach. Nat Rev Neurol $7: 1-13,2011$

12. Lasjaunias P, Piske R, Terbrugge K, Willinsky R: Cerebral arteriovenous malformations (C. AVM) and associated arterial aneurysms (AA): Analysis of $101 \mathrm{C}$. AVM cases, with 37 AA in 23 patients. Acta Neurochir (Wien) 91:29-36, 1988

13. Lv X, Jiang C, Li Y, Yang X, Wu Z: Endovascular treatment for cerebral perforating artery aneurysms. Neurol Res 33:553-557, 2011 
14. Lv X, Li Y, Yang X, Jiang C, Wu Z: Characteristics of arteriovenous malformations associated with cerebral aneurysms. World Neurosurg 76:288-291, 2011

15. Lv X, Wu Z, Jiang C, Yang X, Li Y, Sun Y, Zhang N: Angioarchitectural characteristics of brain arteriovenous malformations presenting with and without hemorrhage. World Neurosurg 76:9599,2011

16. Lv X, Wu Z, Li Y, Jiang C, Yang X, Zhang J: Cerebral arteriovenous malformations associated with flow-related and circle of Willis aneurysms. World Neurosurg 76:455-458, 2011

17. Lv X, Wu Z, Li Y, Yang X, Jiang C, Sun Y, Zhang N: Endovascular treatment of cerebral aneurysms associated with arteriovenous malformations. Eur J Radiol 81:1296-1298, 2012

18. Mandybur TI, Nazek M: Cerebral arteriovenous malformations: A detailed morphological and immunohistochemical study using actin. Arch Pathol Lab Med 114:970-973, 1990

19. Marbacher S, Schläppi JA, Fung C, Hüsler J, Beck J, Raabe A: Do statins reduce the risk of aneurysm development: A case-control study. J Neurosurg 116:638-642, 2012

20. Mast H, Young WL, Koennecke HC, Sciacca RR, Osipov A, Pile-Spellman J, Hacein-Bey L, Duong H, Stein BM, Mohr JP: Risk of spontaneous haemorrhage after diagnosis of cerebral arteriovenous malformation. Lancet 350:1065-8, 1997

21. Meisel HJ, Mansmann U, Alvarez H, Rodesch G, Brock M, Lasjaunias P: Cerebral arteriovenous malformations and associated aneurysms: Analysis of 305 cases from a series of 662 patients. Neurosurgery 46:793-800, 2000

22. Mizutani T, Miki Y, Kojima H, Suzuki H: Proposed classification of nonatherosclerotic cerebral fusiform and dissecting aneurysms. Neurosurgery 45:253-260, 1999
23. Mjoli N, Le Feuvre D, Taylor A: Bleeding source identification and treatment in brain arteriovenous malformations. Interv Neuroradiol 17:323-330, 2011

24. Ondra SL, Troupp H, George ED, Schwab K: The natural history of symptomatic arteriovenous malformations of the brain: A 24-year follow-up assessment. J Neurosurg 73:387-391, 1990

25. Perata HJ, Tomsick TA, Tew JM Jr: Feeding artery pedicle aneurysms: Association with parenchymal hemorrhage and arteriovenous malformation in the brain. J Neurosurg 80:631-634, 1994

26. Redekop G, TerBrugge K, Montanera W, Willinsky R: Arterial aneurysms associated with cerebral arteriovenous malformations: Classification, incidence, and risk of hemorrhage. J Neurosurg 89:539-546, 1998

27. Spetzler RF, Martin NA: A proposed grading system for arteriovenous malformations. J Neurosurg 65:476-483, 1986

28. Stapf C, Mast H, Sciacca RR, Choi JH, Khaw AV, Connolly ES, Pile-Spellman J, Mohr JP: Predictors of hemorrhage in patients with untreated brain arteriovenous malformation. Neurology $66: 1350-1355,2006$

29. Turjman F, Massoud TF, Vinuela F, Sayre JW, Guglielmi G, Duckwiler G: Correlation of the angioarchitectural features of cerebral arteriovenous malformations with clinical presentation of hemorrhage. Neurosurgery 37:856-860, 1995

30. Westphal M, Grzyska U: Clinical significance of pedicle aneurysms on feeding vessels, especially those located in infratentorial arteriovenous malformations. J Neurosurg 92:995-1001, 2000

31. Wilkins RH: Natural history of intracranial vascular malformations: A review. Neurosurgery 16:421-430, 1985 